\title{
PRELIMINARY COMMUNICATION ON THE SHORT-PERIOD OSCILLATIONS OF SOLAR MAGNETIC FIELDS
}

\author{
A. B. Severny \\ (Crimean Astrophysical Observatory, Nauchny, Crimea, U.S.S.R.)
}

With the image of the Sun held fixed, time variations of solar magnetic field were recorded simultaneously in two lines ( $\lambda 5250$ and $\lambda 6103)$ with the aid of double magnetograph (for the description see Severny, 1966, examples of such records are on Figures 1 and 2). The resolution was $2.15 \times 4$ ". 5 . The records in both lines $\lambda 5250$ and $\lambda 6103$ covering a time interval of about 1 hour show oscillations with the period $\sim 7.4 \mathrm{~min}$ and amplitudes 10-20 gauss for moderately active region (full lines on the top of Figure 2). The oscillations of the magnetic field at both levels (the heights of $\lambda 5250$ and $\lambda 6103$ differ by $260 \mathrm{~km}$ ) are sometimes synchronous but now and then phase shift appears. For quiet regions near the centre of the disk the oscillations with the period $\sim 9.0 \mathrm{~min}$ and amplitudes less than 5 gauss are recorded and presented in Figure 1 (full line). These 7-9 min oscillations are probably sometimes damped or interrupted by more short-period fluctuations (with the mean period $80 \mathrm{~s}$ ) of smaller amplitude, but these last oscillations could be due to the fluctuations of seeing.

Simultaneous records of radial velocities (Figure 2, bottom) for both levels at the same time show clearly Leighton's pulsations with the mean period 297 s coinciding with that found in Leighton's (1963) paper. No appreciable changes in intensity (except usual fluctuations due to noises) of both lines recorded simultaneously were found.

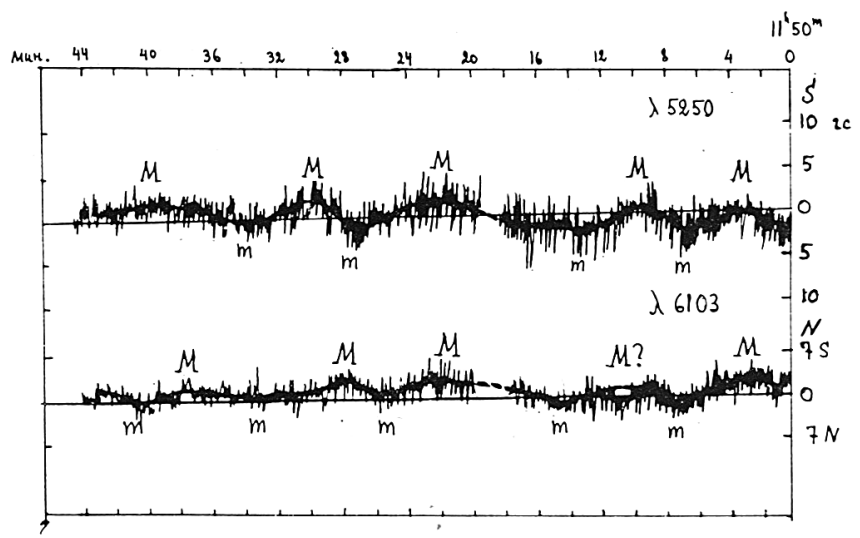

FIG. 1. Fluctuations of magnetic field recorded in 25250 (top) and $\lambda 6103$ (below) for quiet regions near the centre of the disk. 
A. B. SEVERNY

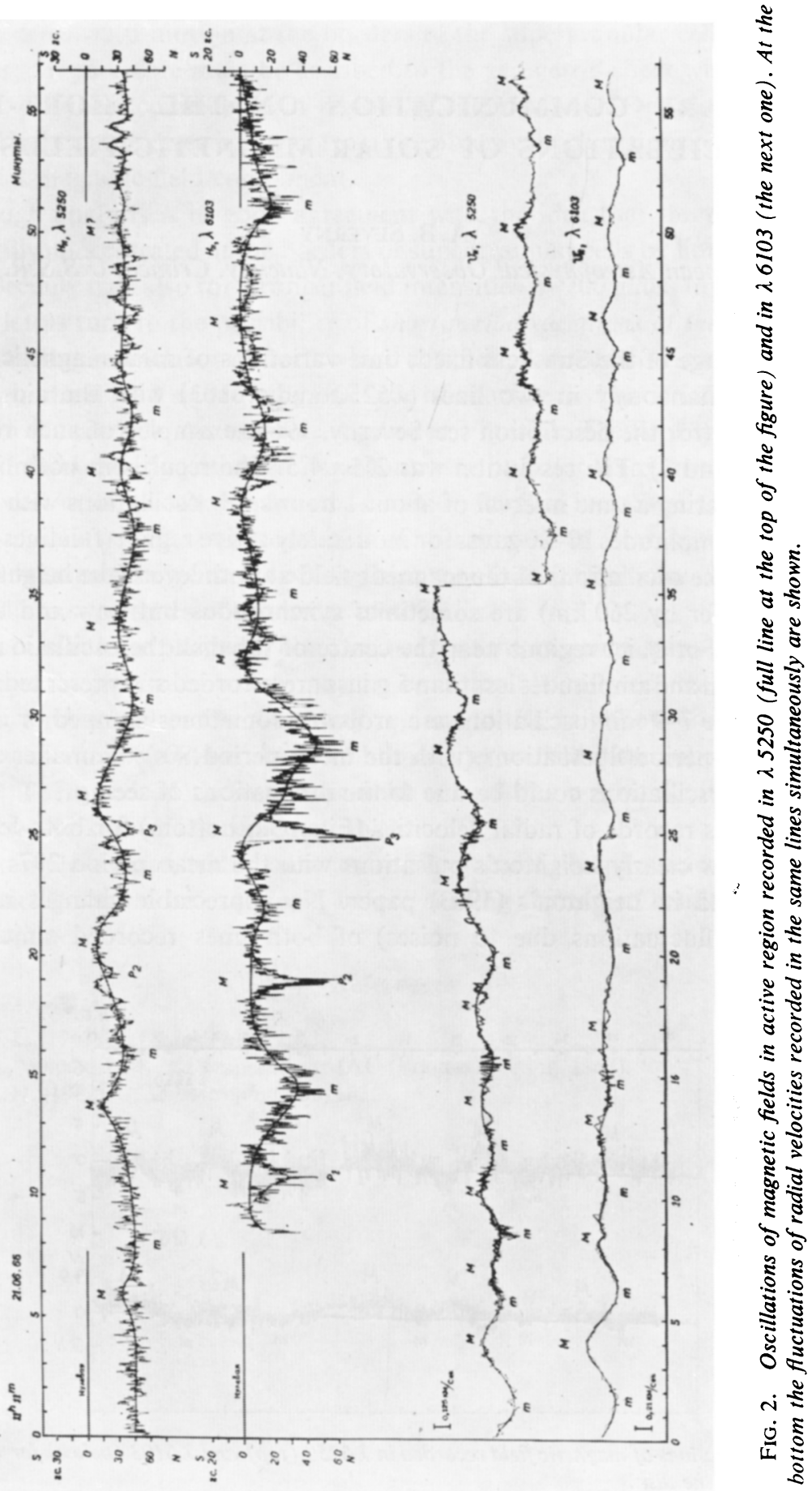




\section{References}

Leighton, R. (1963) A. Rev. Astr. Astrophys., 1, 19.

Severny, A. B. (1966) Astr. Zu., 43, 465.

\section{DISCUSSION}

Howard: Some of the oscillations you showed in the last slide indicate a variation of magnetic field which involved a change in sign of the field. Do you have an explanation for this? How can it happen?

Severny: We have not observed such oscillations in active regions, but in the case of general field (quiet regions) it is difficult to be sure that such changes of sign are reliable due to long-period noises. As regards to the explanation of such oscillations in general neither I nor, I think, Mr. Deubner are in possession of adequate explanation of the physical nature of phenomenon. 